\title{
MiR-93-5p enhances growth and angiogenesis capacity of HUVECs by down-regulating EPLIN
}

\author{
Liang Liang ${ }^{1}$, Lei Zhao ${ }^{2}$, Ying Zan ${ }^{1}$, Qing Zhu ${ }^{1}$, Juan Ren ${ }^{3}$ and Xinhan Zhao ${ }^{4}$ \\ ${ }^{1}$ Department of Oncology, The Second Affiliated Hospital, Medical School of Xi'an Jiaotong University, Xi'an 710004, China \\ ${ }^{2}$ Department of Molecular Physiology and Biophysics, University of Iowa, Carver College of Medicine, Iowa City, IA 52242, \\ USA \\ ${ }^{3}$ Department of Radiation Oncology, The First Affiliated Hospital, Medical School of Xi'an Jiaotong University, Xi'an 710061, \\ China \\ ${ }^{4}$ Department of Oncology, The First Affiliated Hospital, Medical School of Xi'an Jiaotong University, Xi'an 710061, China \\ Correspondence to: Xinhan Zhao, email: zhaoxinhanprof@163.com \\ Juan Ren, email:869491533@qq.com
}

Keywords: miR-93-5p; EPLIN; angiogenesis; HUVECs; TNBC

Received: March 14, $2017 \quad$ Accepted: July 12, $2017 \quad$ Published: November 06, 2017

Copyright: Liang et al. This is an open-access article distributed under the terms of the Creative Commons Attribution License 3.0 (CC BY 3.0), which permits unrestricted use, distribution, and reproduction in any medium, provided the original author and source are credited.

\section{ABSTRACT}

Tumor angiogenesis is essential in delivering oxygen and nutrients to growing tumors, and therefore considered as a hallmark of cancer. MicroRNAs (miRNAs) have been shown to play important roles in regulating tumor angiogenesis. MicroRNA-935p (miR-93-5p) has been identified as an oncogenic miRNA in a variety of human malignancies and involved in tumor angiogenesis in astrocytoma. However, the direct effect(s) of miR-93-5p on the biological behaviors of endothelial cells have not been investigated. Thus, in the present study we investigated the role(s) of miR-93-5p in regulating the functions of human umbilical vein endothelial cells (HUVECs). We found that triple negative breast cancer (TNBC) tissues with higher levels of miR-93-5p showed higher blood vessel density. Overexpression of miR-93-5p accelerated HUVECs proliferation and migration and promoted HUVECs lumen formation and sprouting in vitro, while blockade of miR-93-5p suppressed HUVECs migration and angiogenic capacity. The mechanistic studies revealed that miR-93-5p can promote angiogenic process through inhibiting epithelial protein lost in neoplasm (EPLIN) expression in HUVECs. In sum, our results have indicated that miR-93-5p promoted angiogenesis through down-regulating EPLIN and therefore represented a promising target for developing novel anti-angiogenic therapeutics.

\section{INTRODUCTION}

Tumor angiogenesis is essential in delivering oxygen and nutrients to growing tumors, and therefore considered as a hallmark of cancer [1]. Angiogenesis has been shown to play critical roles in throughout all phases of cancer formation, aggression, invasion and metastasis [2]. Given the importance of angiogenesis in cancer development, in recent years, inhibition of tumor angiogenesis in line with conventional therapies has become an effective anti-cancer strategy against several types of cancers, such as colorectal cancer [3], kidney cancer [4] and liver cancer [5]. Unfortunately, the clinically approved anti-angiogenic reagents are only effective in specific cancer types and in a subset of patients, and many who are initially responsive develop resistance over time [6]. In addition, some primary and metastatic tumors can develop and progress in the absence of angiogenesis, adding another layer of complexity to developing anti-angiogenic therapeutics [6]. Therefore, there is an urgent need to better understand the molecular basis of cancer angiogenesis for developing novel therapeutic strategies against newly formed cancer vasculatures.

MicroRNAs (miRNAs) are a family of short noncoding RNAs that regulate gene expression by binding 
to the 3' untranslated region (3'UTR) of mRNAs [7, 8]. Accumulating evidence has shown that miRNAs are involved in the process of angiogenesis via regulating the expression levels of pro- or anti-angiogenic factors [9]. MiR-93-5p, a member of the miR-106b 25 cluster, has been significantly up-regulated in multiple types of cancers, including breast cancer [10], osteosarcoma [11] and gastric cancer [12], and functions as an oncomiRNA in tumor growth [10-12]. miR-93-5p has also been reported to participate in regulating angiogenesis in cancers. Fang et al. reported that miR-93-5p promoted tumor angiogenesis and metastasis by suppressing LATS2 expression in astrocytoma [13]. In another study, Fang et al. showed that miR-93 promoted astrocytoma growth and angiogenesis by down-regulating integrin- $\beta 8$ [14]. Fabbri et al. reported that miR-93 regulated the secretion of a panel of cytokines, chemokines and growth factors that were involved in angiogenesis in gliomas through suppressing IL-8 and VEGF [15]. However, in these studies, miR-93-5p expression level was either up- or down-regulated in cancer cell lines, which were then co-cultured with endothelial cells (ECs) to examine the effect(s) of miR-93-5p on angiogenic capacities of ECs. No reference regarding the direct effect(s) of miR-93-5p on angiogenesis of ECs has been published.

Epithelial protein lost in neoplasm (EPLIN) is a cytoskeleton-associated protein involved in regulating actin dynamics and cell motility. Sanders et al. showed that overexpression of EPLIN in HECV endothelial cells resulted in a significant reduction in cell migration and tubule formation [16]. In addition, the 3'-UTR of EPLIN mRNA has the targeting sequence of the seed region of miR-93-5p and EPLIN has been experimentally validated as a target of miR-93-5p by several cross-linking immunoprecipitation (CLIP)-based high throughput experiments.

We have reported that miR-93-5p played an oncogenic role in triple negative breast cancer (TNBC) development [17]. Given the pro-angiogenic effect of miR-93-5p in glioma, we therefore investigated whether miR-93-5p was involved in regulating angiogenesis in TNBC and further explored the underlying mechanism(s). In this study, we have demonstrated that TNBC tissues with up-regulated miR-93-5p level also had a higher vessel density. Overexpression of miR-93-5p accelerated human umbilical vein endothelial cells (HUVECs) proliferation and migration and promoted their lumen formation and sprouting in vitro, while blockade of miR93-5p led to suppression in migration and angiogenic response of HUVECs. In addition, the mechanistic studies have revealed that miR-93-5p promoted the angiogenesis process through inhibiting EPLIN expression in HUVECs. In sum, our results have indicated that miR-93-5p promoted angiogenesis through down-regulating EPLIN and represented a promising target for developing novel anti-angiogenesis therapeutics.

\section{RESULTS}

\section{miR-93-5p expression level was positively correlated with vessel density in breast cancer}

Aiming at the role(s) of miR-93-5p in TNBC angiogenesis, we conducted the CD31 staining of the TNBC tissues, and found that the samples with high miR-93-5p expression had more vessels (Figure 1A). Furthermore, we analyzed The Cancer Genome Atlas (TCGA) database for breast invasive carcinoma (BRCA) to find the correlation of miR-93 expression with the expression of blood vessel markers. As shown in Figure 1B, miR-93 expression level had positive correlation with the CD31 and Endomucin (EMCN) expression, which were considered to be strictly expressed in endothelial cells. These results indicated that miR-93-5p might play a vital role in angiogenesis.

\section{miRNA-93-5p enhanced ECs proliferation and migration in vitro}

We then employed in vitro assays to examine the effect(s) of miR-93 overexpression on ECs. HUVECs were transfected with miR-93-5p mimics or control oligos and the overexpression of miR-93-5p was validated by qRT-PCR (Figure 2A). As shown in Figure 2B, up-regulated miR-93-5p levels significantly accelerated the growth of HUVECs. To deplete miR-93$5 \mathrm{p}$, the anti-sense oligo (ASO) of miR-93-5p or control oligo were transfected into HUVECs and inhibition of miR-93-5p was confirmed by qRT-PCR (Figure 2C). The MTT assay revealed that inhibition of miR-93-5p led to decreased cell viability in HUVECs (Figure 2D). To reveal the mechanisms contributing to the increased cell viability induced by up-regulation of miR-93-5p, we examined the alterations in cell cycle. The results indicated that overexpression of miR-93-5p enhanced the entrance of HUVECs into the S/G2/M phases of cell cycle, suggesting miR-93-5p could accelerate the cell cycle progression (Figure 2E). Furthermore, the results of EdU (5-ethynyl-2'-deoxyuridine) incorporation assay indicated that transfection of miR93-5p mimics increased the number of EdU-positive HUVECs compared to cells transfected with control oligos (Figure 2D), suggesting that miR-93-5p also promoted HUVECs proliferations. Endothelial cell migration is essential to angiogenesis. We therefore detected the effect of miR-93-5p on the migration capacity of ECs. The transwell assay indicated that, compared to cells trasfected with control oligos, a larger number of HUVECs trasfected with miR-93$5 \mathrm{p}$ mimics migrated to the lower side of the membrane (Figure 2G), suggesting that miR-93-5p enhanced the migration capacity of HUVECs. In together, these results suggest that miR-93-5p could promote ECs proliferation and migration in vitro. 


\section{miRNA-93-5p promoted ECs lumen formation and angiogenic sprouting in vitro}

HUVECs were transfected with miR-93-5p mimics, ASO and corresponding negative controls, respectively, and subjected to the lumen formation assay. The results showed that miR-93-5p overexpression increased the number and the length of branches (Figure 3A), while blockade of miR93-5p inhibited the HUVECs lumen formation in terms of both number and length on the matrigel matrix (Figure 3B). We further detected the effects of miR-93-5p on angiogenic sprouting capacity of HUVECs by performing a fibrin gel beads sprouting assay. HUVECs transfected with miR-93$5 \mathrm{p}$ mimics formed a larger number of sprouts with longer length compared to the control cells (Figure 3C). However, miR-93-5p inhibitory oligos suppressed the angiogenic sprouting by decreasing both the number and the length of sprouts (Figure 3D). In together, these results indicated that miR-93-5p could promote angiogenesis in vitro.

\section{EPLIN was a direct target of miR-93-5p}

EPLIN is a cytoskeleton-associated protein involved in regulating actin dynamics and cell motility. Sanders et al. showed that over-expression of EPLIN in HECV endothelial cells resulted in a significant reduction in cell migration and tubule formation [16]. The sequence of 3'UTR of EPLIN mRNA is recognizable by the seed region of miR-93-5p (Figure 4A). EPLIN has been predicted as a target of miR-93-5p by multiple bioinformatics tools, including TargetScan, microRNA.org and miRDB. In addition, EPLIN has been experimentally validated as a target of miR-93-5p by several CLIP-based high throughput experiments. To establish the regulatory association between miR-93-5p and EPLIN, we initially measured the expression levels of EPLIN in response to changes in miR-93-5p levels. The results of qRT-PCR revealed that transfection of miR-93-5p mimics reduced the mRNA level of EPLIN, while inhibition of miR-93$5 \mathrm{p}$ by transfection with ASO up-regulated EPLIN mRNA levels (Figure 4B). The western blots assay further confirmed this result, showing that transfection of miR93-5p mimics and ASO led to reduction and increase in EPLIN protein levels, respectively (Figure 4C). To investigate whether EPLIN was a direct target of miR-93$5 \mathrm{p}$, we constructed luciferase reporters containing the 3 'UTR sequence of EPLIN mRNA with either the wildtype or the mutant seed region of miR-93-5p (Figure 4A). The
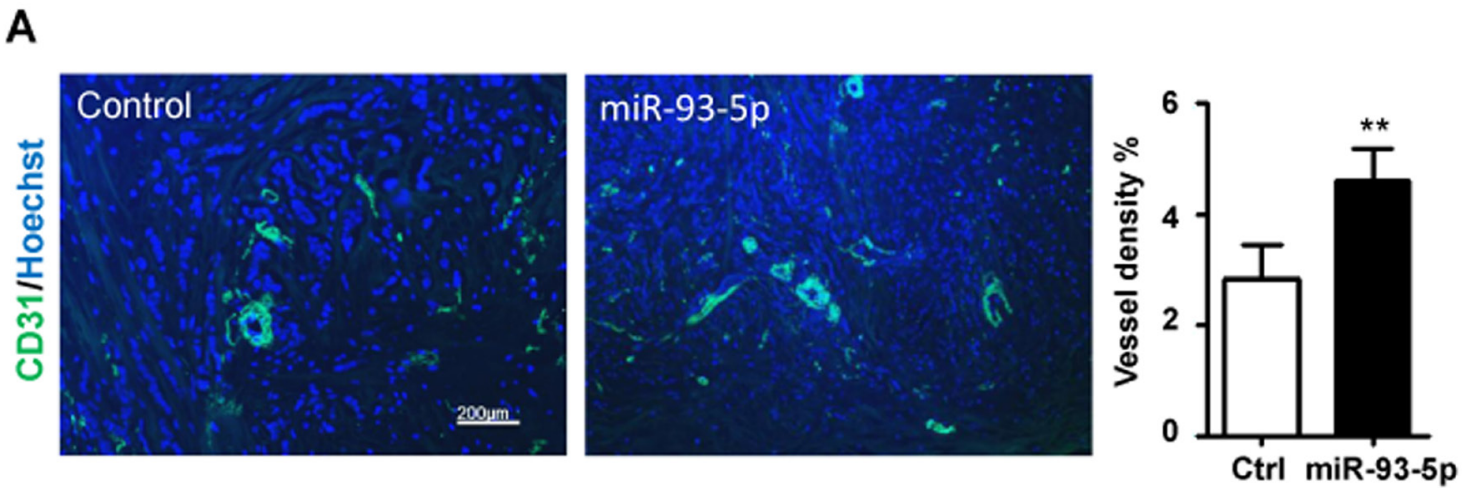

B
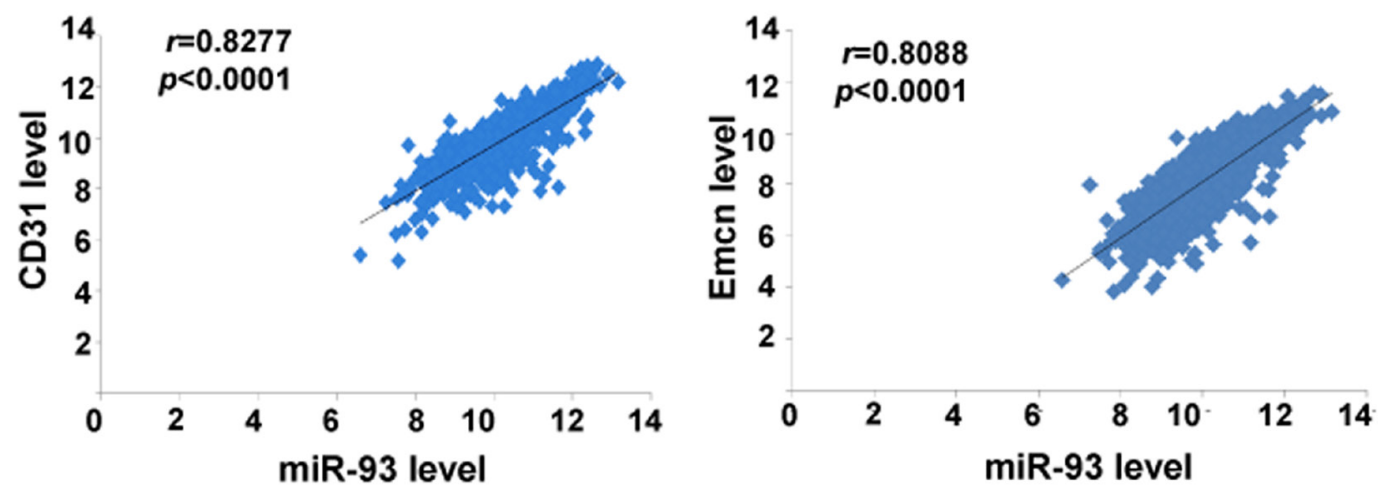

Figure 1: miR-93-5p expression level was positively correlated with blood vessel density in breast cancer. (A) TNBC tissues were stained for CD31 and Hoechst, then analyzed by a fluorescence microscope, and the percentages of area with positive CD31 were calculated. Bars $=$ means $\pm \mathrm{SD}(\mathrm{n}=4),{ }^{* *}, P<0.01$. (B) The BRCA dataset from the TCGA database was downloaded, and the expression levels of miR-93, CD31 and Emcn in each sample was recorded and analyzed for correlations. 
A

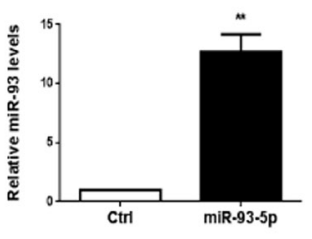

C

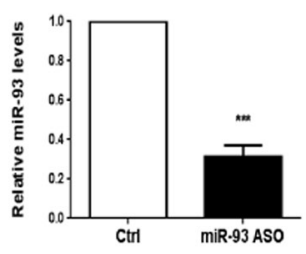

B

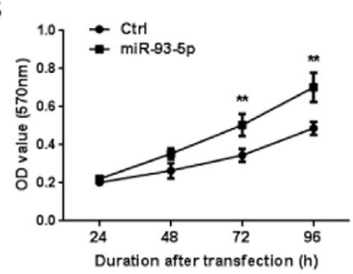

D

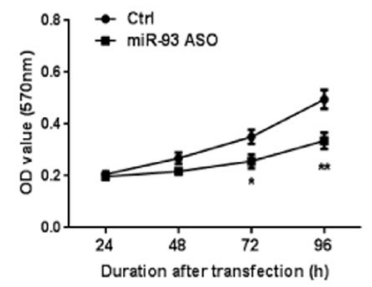

E
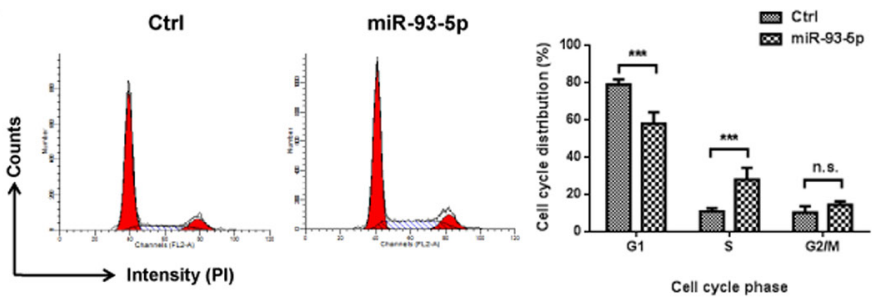

$\mathbf{F}$

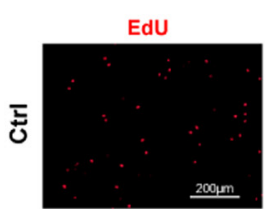

EdU/Hoechst
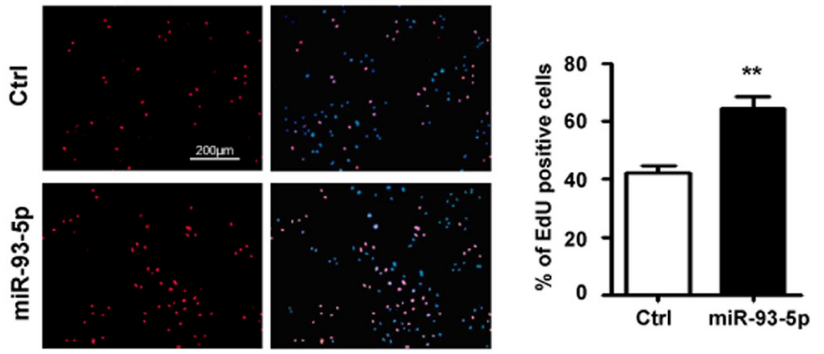

G
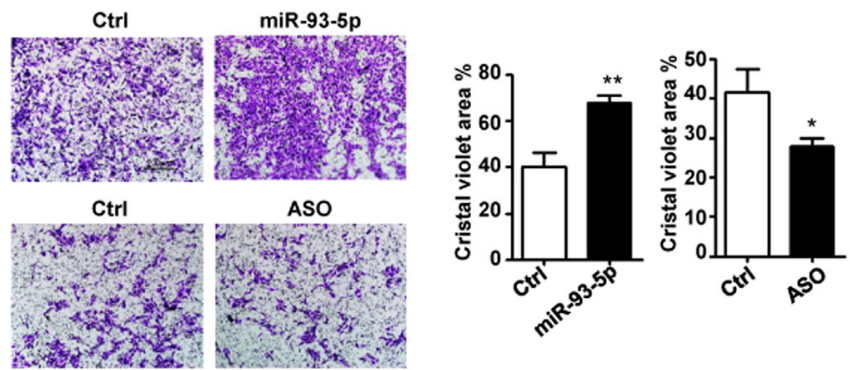

Figure 2: miR-93-5p enhanced ECs proliferation and migration in vitro. (A) and (B) HUVECs were transfected with $100 \mathrm{nM}$ miR-93-5p mimics or control oligos. (A) Up-regulation of miR-93-5p was confirmed using qRT-PCR. (B) Cell viability was determined by the MTT assay. (C and D) HUVECs were transfected with $100 \mathrm{nM}$ miR-93-5p ASO or control oligos. (C) qRT-PCR was performed to validate knock-down of miR-93-5p. (D) Cell viability was determined by the MTT assay. (E) HUVECs were transfected with 100 nM miR-93-5p or control oligos. Cell cycle distribution was analyzed by using PI staining flow cytometry. Left panel: representative histograms. Right panel: quantification of percentage of cells at each phase of a cell cycle. (F) HUVECs were transfected with $100 \mathrm{nM}$ miR-93-5p mimics or ASO and corresponding control oligos. An EdU incorporation assay was performed using a fluorescence microscope. The percentage of proliferating cells was defined as the ratio of EdU positive cells to total cells determined by the Hoechst staining. Left panel: representative florescent images. Right panel: quantification of the percentage of EdU positive cells. (G) HUVECs were transfected as described in (F). The capacity of cell migration was measured using a transwell assay. Cells that transmigrate to the lower side of the chamber were stained and quantified. Top panel: representative figures. Bottom panel: quantification of areas stained by crystal violet over total areas. All bars $=$ means $\pm \mathrm{SD}$ of at least three independent experiments, ${ }^{*}, P<0.05,{ }^{* *}, P<0.01$. 
reporter assay further confirmed that miR-93-5p repressed EPLIN expression by directly combining with the 3'-UTR of EPLIN mRNA (Figure 4E), and this inhibitory effect was eliminated when the recognition site was disrupted by mutations (Figure 4E).

\section{miR-93-5p promoted cell migration and angiogenesis through targeting EPLIN}

We further investigated whether the effects of miR-93-5p on HUVECs migration and angiogenesis was through down-regulating EPLIN. A siRNA specifically targeting EPLIN led to decreased mRNA and protein levels of EPLIN in HUVECs (Figure 5A and 5B). We also found that depletion of EPLIN by transfection of specific siRNA could attenuate the effects of miR-93-5p ASO on the ECs migration (Figure 5C) and lumen formation (Figure 5D), further confirming that miR-93-5p could regulate angiogenesis by directly targeting the EPLIN transcripts.

\section{DISCUSSION}

Tumor angiogenesis is essential for almost all steps in cancer formation, aggression, and metastasis and thus has been considered as a hallmark of cancer [1]. Antiangiogenesis has become an effective anti-cancer strategy in several types of cancers, such as colorectal cancer,
A
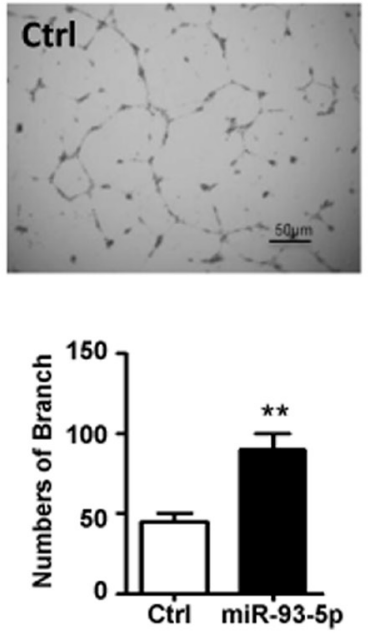

C
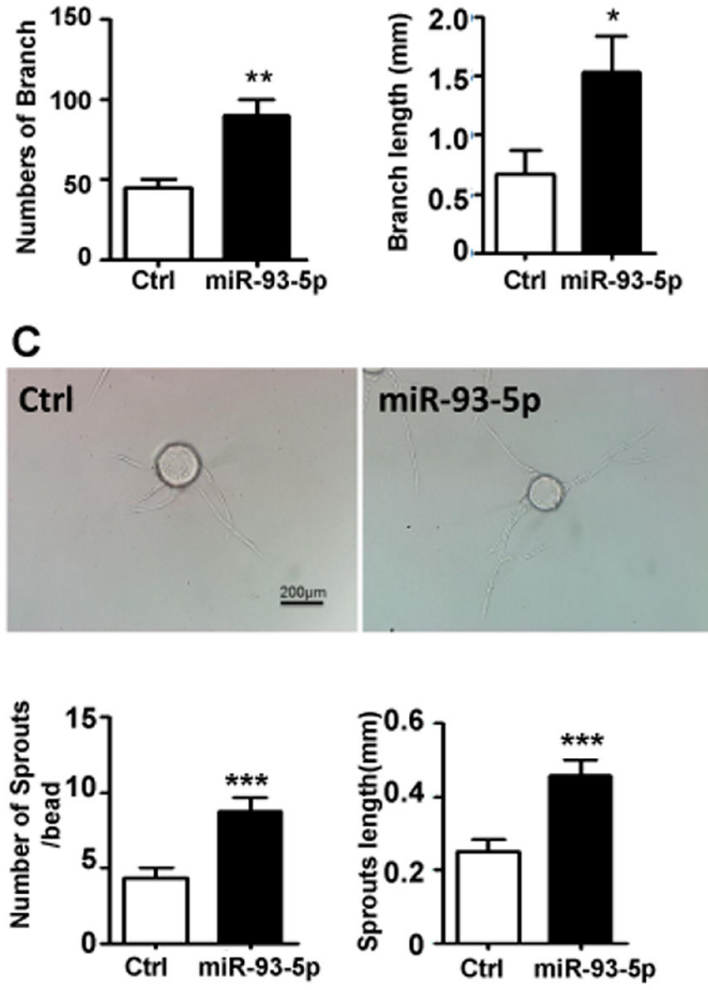

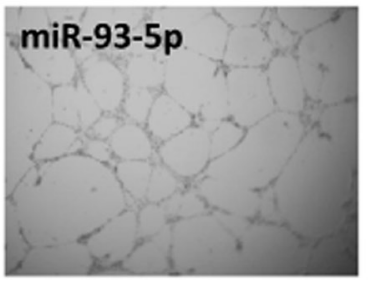

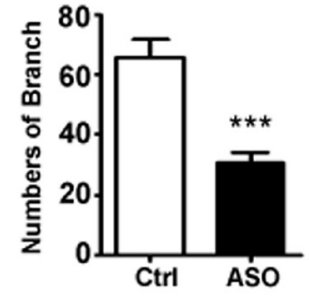

D
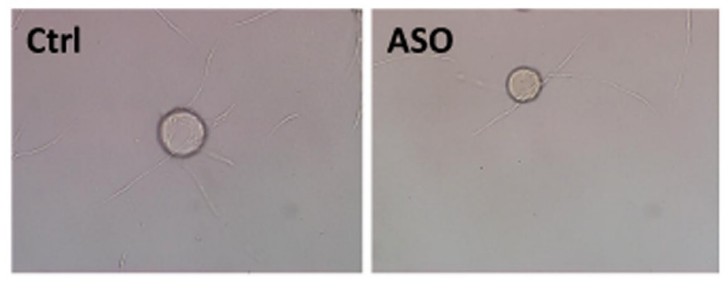

B
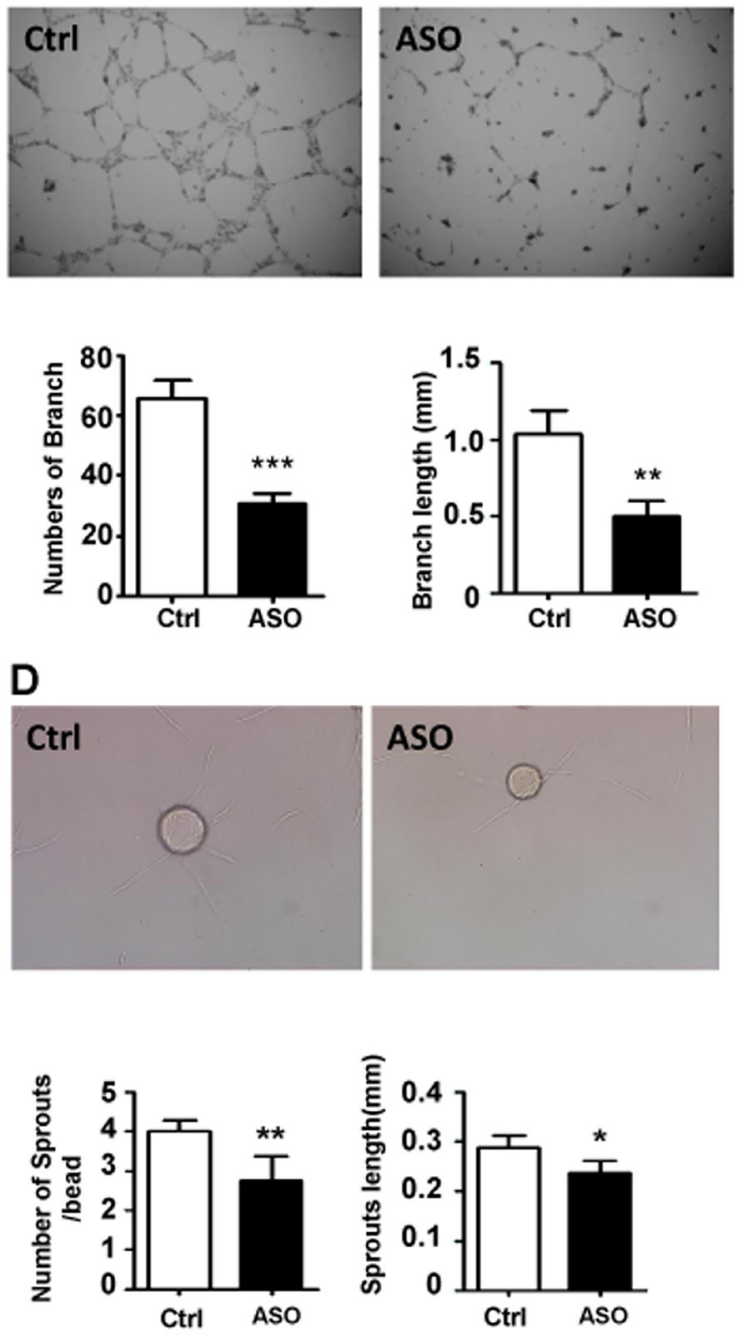

Figure 3: miR-93-5p promoted ECs lumen formation and sprouting in vitro. (A and B) HUVECs were transfected with miR-93-5p mimics (A), miR-93-5p ASO (B), or corresponding control oligos; 48h after transfection the cells were digested and seeded on the matrigel. The number and length of branches were analyzed and compared between the two groups. Top panel: representative figures. Bottom panel: quantified number and length of branches. (C and D) HUVECs were transfected as described above. Cells were trypsinized $24 \mathrm{~h}$ after the transfection and tested for sprouting capacity with a fibrin gel assay. Images were captured and the number and length of sprouts were calculated and compared between the two groups. Top panel: representative figures. Bottom panel: quantified number and length of sprouts. All bars $=$ means $\pm \mathrm{SD}$ of 4 independent experiments, ${ }^{*}, P<0.05,{ }^{* *}, P<0.01,{ }^{* * *}, P<0.001$. 


\section{A}

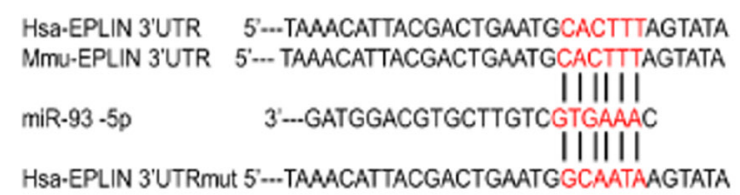

B
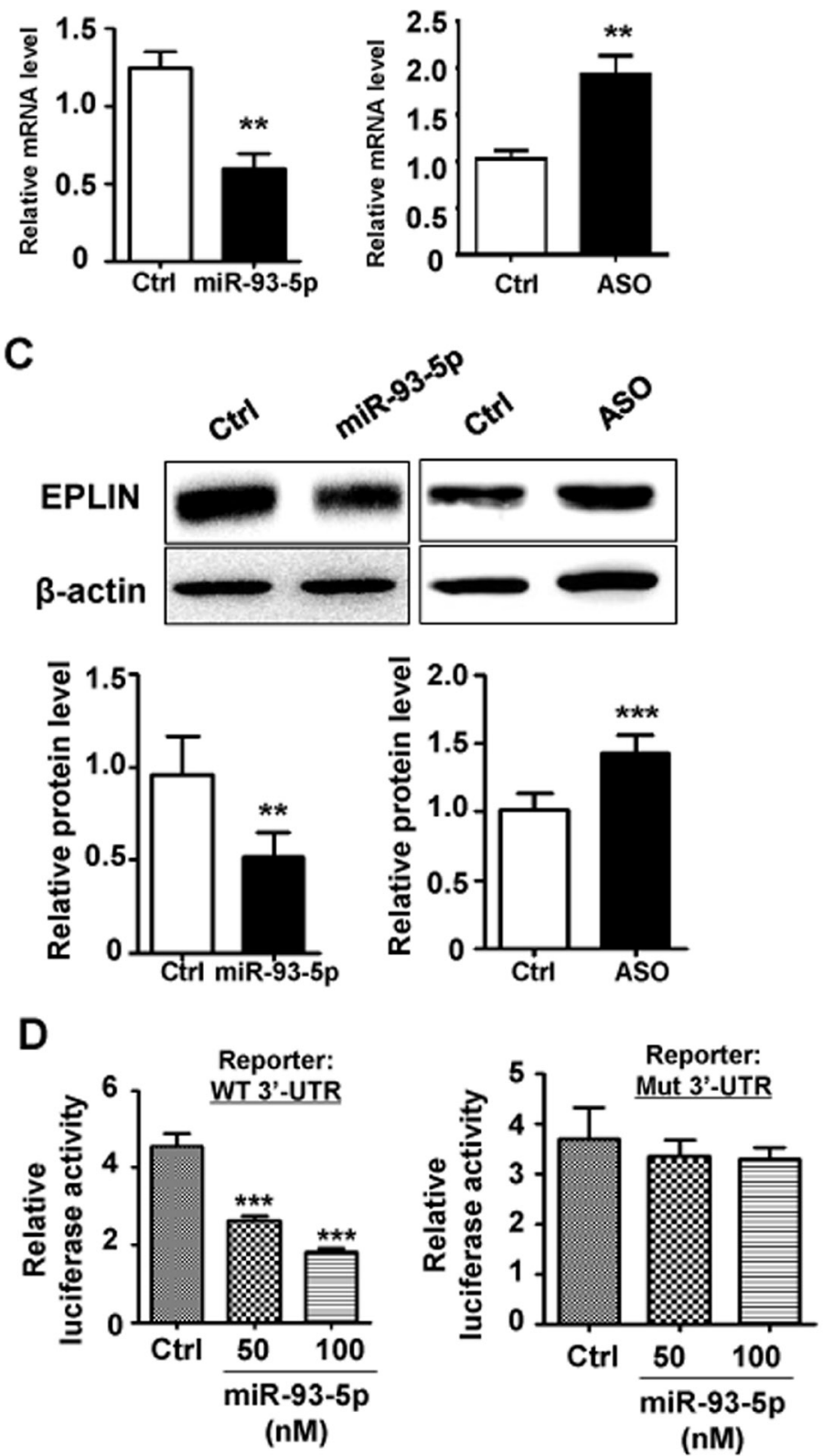

Figure 4: EPLIN was identified as a direct target of miR-93-5p. (A) the 3' UTR of human and mouse EPLIN mRNA were aligned with miR-93-5p. The complementary sequence was marked in red. The 3' UTR of human EPLIN mRNA with mutated miR-93-5p targeting sequence was also shown. (B) HUVECs were transfected with miR-93-5p mimics (left panel), miR-93-5p ASO (right panel), or corresponding controls. The changes in the mRNA level of EPLIN were detected by qRT-PCR. (C) HUVECs were transfected as described in (B), and the protein levels of EPLIN were determined by a western-blot assay. Bands were quantitatively compared between groups. (D) HeLa cells were cotransfected with $100 \mathrm{ng}$ of pGL3-EPLIN-WT or pGL3-EPLIN-mt, together with miR-93-5p mimics (50, $100 \mathrm{nM})$ or control oligos, and pRL-TK plasmid ( $5 \mathrm{ng}$ ). Cells were collected $48 \mathrm{~h}$ after transfection, and the luciferase activity was analyzed. All bars $=$ means $\pm \mathrm{SD}$ of at least 3 independent experiments, ${ }^{*}, P<0.05,{ }^{* *}, P<0.01,{ }^{* * *}, P<0.001$. 
kidney cancer and liver cancer [2, 4-6]. Unfortunately, the anti-angiogenic reagents are effective only in specific cancer types and drug resistance develops over time. Therefore, a better understanding of the molecular basis of tumor angiogenesis is critical for developing novel therapeutic strategies and reversing drug resistance.

Recent publications demonstrated that miR-93-5p is frequently up-regulated in multiple types of cancers, including breast cancer [10], osteosarcoma [11] and gastric cancer [12] and functions as an onco-miRNA in tumor growth [10-12]. Accumulating evidence suggests that miRNAs participate in modulating angiogenesis through regulating the expression levels of a variety of angiogenesisrelated genes [9]. However, the role(s) of miR-93-5p in regulating angiogenesis remain largely underexplored. In this study, we demonstrate that the TNBC samples with high
miR-93-5p expression had higher levels of blood vessels marker CD31 staining (Figure 1A), suggesting miR-93-5p might be involved in angiogenesis regulation. This result was consistent with the findings in gliomas [13-15]. Fang et al. reported that miR-93-5p promoted tumor angiogenesis and metastasis by suppressing LATS2 expression in astrocytoma [13]. In another study, Fang et al. showed that miR-93 promoted astrocytoma growth and angiogenesis by downregulating integrin- $\beta 8$ [14]. Fabbri et al. reported that miR-93 regulated the secretion of a panel of cytokines, chemokines and growth factors that were involved in angiogenesis in gliomas through suppressing IL-8 and VEGF [15]. However, these assays were performed based on the co-culture of tumor cells ectopically over-expressing miR-93-5p with endothelial cells. No literature has reported the role(s) of miR-93-5p in regulating the angiogenic capacity of endothelial cells
A

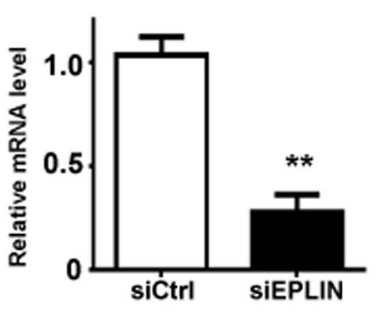

C

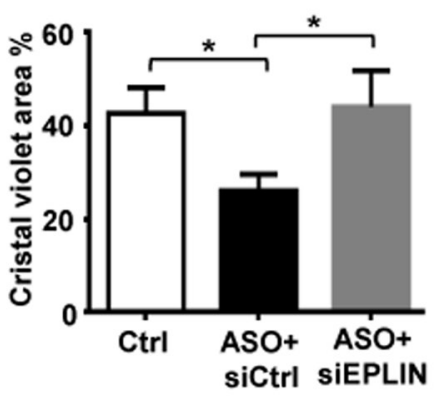

B

EPLIN

$\beta$-actin

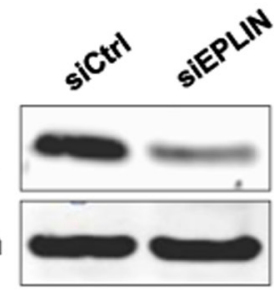

D

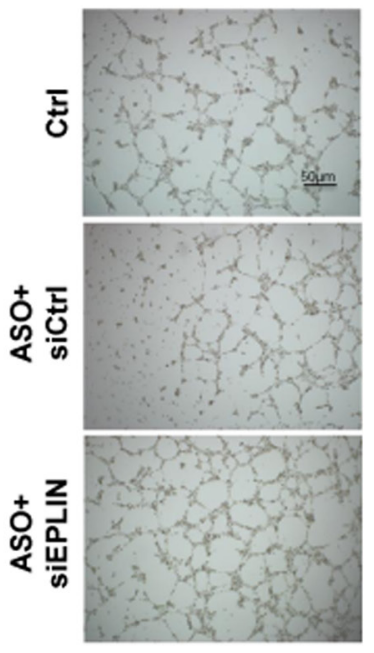

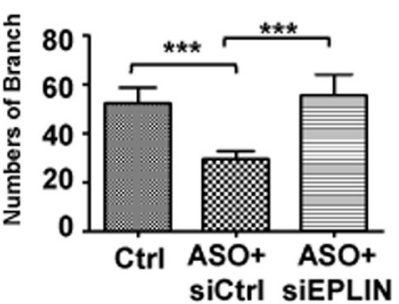

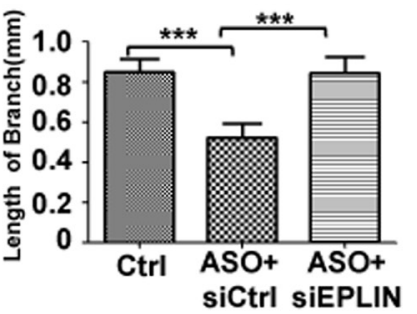

Figure 5: miR-93-5p Promoted Cell Migration and Angiogenesis through Targeting EPLIN. (A and B) HUVECs were transfected with $50 \mathrm{nM}$ EPLIN specific siRNA or non-targeting siRNA for 48h. (A) The mRNA levels of EPLIN were measured using qRT-PCR. (B) Protein levels of EPLIN were determined using an immunoblotting assay. (C and D) HUVECs were transfected with control oligos, miR-93-5p ASO plus non-targeting siRNA, or miR-93-5p ASO mixed with siEPLIN. (C) The cell migration capacity was analyzed using a transwell assay. (D) Cells were seeded on the matrigel for lumen formation assay. Images were captured and the number and length of branches were quantitatively compared among groups. All bars $=$ means $\pm \mathrm{SD}$ of 3 independent experiments, ${ }^{*}, P<0.05,{ }^{* *}, P<0.01$, ***, $P<0.001$. 
directly. Therefore, we investigated the direct role(s) of miR93-5p in regulating the angiogenesis capacity of HUVECs.

Initially, we investigated the effects of miR-93-5p on HUVECs growth in vitro. We found that up- and downregulated miR-93-5p levels significantly accelerated and decreased the growth of HUVECs, respectively (Figure $2 \mathrm{~B}$ and 2D). Further studies revealed that over-expression of miR-93-5p enhanced cell cycle progression into the S/G2/M phases (Figure 2E) and promoted HUVECs proliferations (Figure 2F). The transwell assay indicated that miR-93-5p enhanced the migration capacity of HUVECs, which is essential to angiogenesis (Figure 2G). These functions were in consistent with the oncogenic functions of miR-93-5p in cancer cells, including promoting tumor growth and cell cycle progression. We then investigated the effects of miR-93-5p on blood vessel generation by measuring HUVECs lumen formation and angiogenic sprouting in vitro. The results revealed that miR-93-5p overexpression increased the number and length of branches (Figure 3A), while suppression of miR-93-5p inhibited the HUVECs lumen formation in terms of both number and length on the matrigel matrix (Figure 3B). Furthermore, the fibrin gel beads sprouting assay revealed that miR-93-5p promoted the angiogenic sprouting capacity of HUVECs by increasing the number and length of sprouts (Figure 3C). In together, these results indicated that miR-93-5p could promote angiogenesis in vitro. These results were in consistence with the co-culture based studies in gliomas as mentioned above. However, to further confirm the results of in vitro studies, animal-based in vivo experiments are guaranteed in further studies.

MiRNAs exert functions through regulating target mRNA transcripts. As a candidate target of miR-93-5p, ELPIN was down-regulated by miR-93-5p which bonded to its target site within the 3'UTR of ELPIN (Figure 4). EPLIN is a cytoskeleton-associated protein encoded by the LIMA1 gene, involved in regulating actin dynamics by cross-linking and stabilizing filaments and is involved in regulation of cell motility [18]. EPLIN expression is often decreased in a variety of cancers, including breast cancer, prostate cancer and esophageal cancer [18]. The loss of EPLIN may contribute to the increased invasive phenotype in cancer cells. Not surprisingly, EPLIN also appeared to show an anti-angiogenic role in vitro. Sanders et al. showed that over-expression of EPLIN in HECV endothelial cells resulted in a significant reduction in cell migration and tubule formation [16]. Interestingly, ELPIN was also proved to stabilize vascular capillary network in vitro by interacts with $\alpha$-catenin and actin filaments in endothelial cells. With this characteristic, endothelial cells depletion of ELPIN exhibited a reduced capacity to form pseudocapillary networks and more leakage. We found that depletion of EPLIN by transfection of specific siRNA could attenuate the inhibitory effect of miR-93-5p ASO on the HUVECs migration (Figure 5C) and lumen formation capacities (Figure 5D), further confirming that miR-93-5p could promote angiogenesis by directly targeting EPLIN. These results suggested that miR-93-5p might enhance angiogenesis and reduce vascular capillary stabilization by targeting ELPIN and that both miR-93-5p and EPLIN represented promising targets for developing novel antiangiogenic therapeutics. Notably, a specific miRNA usually targets a variety of mRNA transcripts; therefore, in addition to EPLIN, other target genes of miRN-93$5 \mathrm{p}$ also have been reported, such as $\mathrm{p} 21, \mathrm{E} 2 \mathrm{~F}-1$, PTEN, CCNB1 and LATS2 [13, 19-21]. Moreover, some of the miRN-93-5p targets, such as LATS2, are associated with the angiogenic process. Thus, investigations in the role(s) of other potential targets of miRN-93-5p in the angiogenic capacity of ECs are guaranteed in our future studies.

In sum, our findings first demonstrated that miR-93$5 \mathrm{p}$ enhanced angiogenesis and reduced vascular capillary stabilization by targeting ELPIN in HUVECs. Further studies are guaranteed to fully understand the roles of miR$93-5 p$ in regulating tumor angiogenesis, which include the expression levels of miR-93-59 in endothelial cells of tumor blood vessels and animal model-based in vivo studies. We believe that this study contributed to the understanding of miR-93-5p's biological functions, shed new light on the regulation of angiogenesis and provided a potential novel target for developing anti-angiogenic reagents.

\section{MATERIALS AND METHODS}

\section{Human tissues}

Human TNBC tissues and human umbilical cord biopsies were collected from the Department of Oncology and the Department of Obstetrics and Gynecology, respectively, of the Second Affiliated Hospital Xi'an Jiaotong University. All human participants in the study had signed informed consent for the use of their tissue samples according to the protocols approved by the Ethics Review Board at the Medical School of Xi'an Jiaotong University.

\section{Analysis of the BRCA dataset in TCGA database}

The BRCA dataset for 1097 invasive breast cancer samples was obtained from TCGA. Level 3 miRNASeq data for miR-93 and mRNA-seq data for CD31 and EMCN were retrieved from the TCGA Data Portal. The correlations between the expression of miR-93 and CD31 or EMCN were calculated using the Pearson's correlation analysis. As the data were obtained from TCGA, further approval by an ethics committee was not required. This study meets the publication guidelines provided by TCGA.

\section{Cell culture and transfection}

Primary HUVECs were isolated and characterized by staining with CD31 and photographing under a fluorescence microscope as described previously [22]. 
The purity of HUVECs was close to $95 \%$ (Supplementary Figure 1). HUVECs were maintained in the EC medium (ScienCell, USA) supplemented with 5\% FBS and EC growth supplements at $37^{\circ} \mathrm{C}$ in a humidified atmosphere containing $5 \% \mathrm{CO}_{2}$. Cells at passages between 2 and 5 were used in all experiments. HUVECs were transfected with $100 \mathrm{nM}$ of miR-93-5p mimic oligos (RiboBio, China) or miR-93-5p ASO (RiboBio, China) by using Lipofectamin 2000 reagent (Invitrogen, USA) according to manufacture's instructions. HUVECs transfected with respective non-targeting oligos were used as controls. HeLa cells were obtained from Peking Union Medical Center Laboratory (Beijing, China) and cultured in Dulbecco's modified Eagle's medium (DMEM) supplemented with $10 \%$ fetal calf serum $(\mathrm{FCS})$ at $37^{\circ} \mathrm{C}$ in a humidified atmosphere containing $5 \% \mathrm{CO}_{2}$.

\section{The MTT assay}

The MTT assay was performed to measure cell viability as described before [23]. After transfection, HUVECs were inoculated in 96-well plates at 2500 cells/ well in quadruplicate and incubated at $37^{\circ} \mathrm{C}$. At different time points $(24,48,72$ or $96 \mathrm{~h}), 10 \mu \mathrm{MTT}$ solution (5 $\mathrm{mg} / \mathrm{mL}$; Sigma, USA) was added to each well, and the plates were incubated at $37^{\circ} \mathrm{C}$ for another $4 \mathrm{~h}$. Then, 100 $\mu \mathrm{SDS}-\mathrm{HCl}(10 \%)$ solubilization buffer was added to each well and incubated for overnight. The absorbance at $570 \mathrm{~nm}$ wavelength was measured by using a microplate reader. Three independent experiments were performed for each treatment.

\section{Cell cycle analysis}

HUVECs were trypsinized, washed with icecold PBS, and fixed in $75 \%$ ethanol at $4{ }^{\circ} \mathrm{C}$ for at least 2 hrs. The collected cells were treated in PBS containing $0.02 \%$ Triton $\mathrm{X}-100,3000 \mathrm{U} / \mathrm{mL}$ RNase A and $50 \mu \mathrm{g} / \mathrm{mL}$ propidium iodide for 30 minutes at $37^{\circ} \mathrm{C}$. Subsequently, the cell cycle was investigated with flow cytometry by using a FACSCalibur flow cytometer (BD Immunocytometry Systems, USA). Data analyses were performed using the ModFit LT software (Verity Software House, USA).

\section{Cell proliferation assay}

Cell proliferation was detected by using the CellLight EdU proliferation assay kit (RiboBio, China) according to manufacture's instructions. Briefly, HUVEC cells were cultured in EC medium containing $50 \mu \mathrm{M}$ Apollo 567-conjugated EdU (5-ethynyl-20-deoxyuridine) for $2 \mathrm{hrs}$ and then fixed with 4\% paraformaldehyde for 20 min. The fluorescent signal was detected and captured by an Olympus BX51 fluorescence microscopy (Olympus, Japan). More than 5 random fields per well were captured at a magnification of 100 and the percentage of EdU- positive cells was calculated as Apollo 567 fluorescence positive cells in total cells identified by Hoechst 33342 nuclear staining.

\section{Cell migration assay}

Confluent HUVECs monolayers were trypsinized and seed in the Transwell chamber (Millipore, USA) placed in 24-well plates and cells were cultured to migrate to the lower side of the polycarbonate membrane in complete medium for $24 \mathrm{hrs}$. Cells on the lower side were fixed with $4 \%$ paraformaldehyde and stained with crystal violet and the number of stained cells was counted under a microscope.

\section{Endothelial lumen formation assay}

For the lumen formation assay, 48 well plates were pre-coated with $200 \mu \mathrm{l}$ of the Matrigel Basement Membrane Matrix (BD Biosciences, USA). HUVECs were then seeded on the wells and cultured at $37^{\circ} \mathrm{C}$. After 8 hours, images were photographed under a microscope and the number of branches and the length of the cell cords of the enclosed lumens were determined.

\section{Fibrin gel beads sprouting assay}

Fibrin beads sprouting assay was conducted according to the standard protocol. Briefly, HUVECs were digested and incubated with the Cytodex 3 microcarrier beads (Sigma, USA) at a density of 400 cells per bead at $37^{\circ} \mathrm{C}$ for overnight. The microbeads were then mixed with fibrinogen (Sigma, USA) containing $0.625 \mathrm{U} / \mathrm{ml}$ thrombin (Sigma, USA) at a density of 100 beads $/ \mathrm{ml}$ and embedded in a 48 -well plate, and $0.5 \mathrm{ml}$ of EGM-2 medium (Clonetics, USA) containing with lung fibroblasts (20,000 cells/well) was added. The medium was changed every other day for 4 days. Images of the beads were captured under a microscope (CKX41, Olympus, Japan), and the number and length of sprouts were measured.

\section{Western blot analysis}

Western blots was performed as previously described [24]. Cells were lysed in the RIPA buffer (Beyotime, China), and the protein concentration was measured by using a BCA Protein Assay kit (Pierce, USA). Totally $30 \mu \mathrm{g}$ cell lysates were separated by sodium dodecyl sulfate-polyacrylamide gelelectrophoresis (SDSPAGE), blotted onto polyvinylidene fluoride (PVDF) membranes, which were blocked with 5\% non-fat dried milk in TBS-T and then probed with primary antibodies. Then the membranes were incubated with horseradish peroxidase (HRP)-conjugated goat anti-rabbit IgG or goat anti-mouse IgG (Boster Bio Tec, China). $\beta$-actin was used as a loading control. The primary antibodies included rabbit anti-human EPLIN (1:250, Abcam, USA), mouse 
anti- $\beta$-actin (1:1000, Sigma, USA). Membranes were then detected by using the enhanced chemoluminescence (ECL) system (Clinx Science Instruments, China).

\section{qRT-PCR for EPLIN mRNA levels}

The qRT-PCR was performed as previously described [25]. Total RNA was extracted with the TRIzol reagent (Invitrogen, USA) according to the manufacturer's protocol. Briefly, $1.0 \mu \mathrm{g}$ total RNA was reverse transcribed into cDNA using a PrimeScript RT Reagent Kit (Takara Bio, China) in a $20 \mu 1$ reaction system according to the manufacturer's protocol. Real-time PCR was conducted by using a SYBR Premix Ex Taq Kit (Takara Bio, China) on an ABI PRISM 7500 Real-time PCR system (Life Technologies, USA). qRT-PCR cycling conditions were $95{ }^{\circ} \mathrm{C}$ for $30 \mathrm{sec}$, followed by 40 cycles of $95^{\circ} \mathrm{C}$ for $5 \mathrm{sec}$ and $60^{\circ} \mathrm{C}$ for 30 sec. Glyceraldehyde-3-phosphate dehydrogenase (GAPDH) as used as an internal control. The fold-change in EPLIN levels was calculated using the $2-{ }^{\Delta \Delta} \mathrm{CT}$ method. The PCR primers are listed in Supplementary Table 1.

\section{qRT-PCR for miR-93 expression}

Total RNA, including microRNAs, was extracted using a miRNeasy mini kit (Qiagen, USA) according to manufacture's instructions. The total RNA was converted to cDNA and added with a universal tag by using a miScript II RT kit (Qiagen, USA). To measure the levels of miR-93, a qRT-PCR assay was conducted via using the miScript SYBR Green PCR kit (Qiagen, USA) according to manufacture's instructions. The following primers were used: miR93, 5'-CAAAGTGCTGTT CGTGCAGGTAG -3' and U6 5'-GGATGACACGCAAATTCGTGAAGC -3'. The PCR protocol included $95{ }^{\circ} \mathrm{C}$ for $15 \mathrm{~min}$, followed by 40 cycles of $94{ }^{\circ} \mathrm{C}$ for $15 \mathrm{sec}, 55^{\circ} \mathrm{C}$ for $30 \mathrm{sec}$, and 70 ${ }^{\circ} \mathrm{C}$ for $30 \mathrm{sec}$. The qRT-PCR was performed in triplicate. The expression level of miR-93 was normalized to U6 snRNA. The fold-change in miR-93 levels was calculated using the $2{ }^{\Delta \Delta} \mathrm{C}$ T method.

\section{Luciferase reporter assay}

The 3'-UTR of the human EPLIN mRNA (NM_001114676.1) was amplified from cDNA derived from total RNA of HUVECs, and subcloned into pGL3-promoter vector (Promega, USA) to construct a pGL3-EPLIN-WT reporter plasmid. A mutant reporter (pGL3-EPLIN-mt) with a mutation in the 3'-UTR complementary to the seed sequence of miR-93-5p was generated by using a Site Directed Mutagenesis Kit (Clontech, USA) according to manufacture's instructions. The PCR primers are listed in Supplementary Table 1. To perform the luciferase reporter assay, HeLa cells were cotransfected with 100 ng of pGL3-EPLIN-WT or pGL3EPLIN-mt, together with miR-93-5p mimics $(50,100$
$\mathrm{nM}$ ) or control oligos, and pRL-TK (5 ng). Cells were collected $48 \mathrm{~h}$ after the transfection, and firefly and renilla luciferase activities were analyzed with the Dual-Luiferase Reporter Assay System (Promega, USA) according to the manufacturer's instruction.

\section{Immunofluorescence}

HUVECs were grown on a cover slide until confluent and then fixed with $4 \%$ paraformaldehyde. Tumor cryosections or the fixed HUVECS were blocked and permeabilized in PBS containing $1 \%$ bovine serum albumin (BSA) and $0.3 \%$ TritonX-100, then immunostained with rat anti-CD31 (1:400, BD, USA) antibody at $4{ }^{\circ} \mathrm{C}$ for overnight. After washing with PBS, sections were incubated with FITC-conjugated goat antirat secondary antibody(1:500, Abcam, USA) at room temperature for 2 hours, followed by Hoechst staining. Images were captured with a fluorescence microscope.

\section{Statistics}

The Image Pro Plus 6.0 was used to quantify images. All data analysis was performed using Graph Pad Prism5 software (GraphPad Software, USA). Student's $t$ test was used to compare means of two groups. The one-way ANOVA followed by Tukey post hoc analysis was carried out to compare means of more than two groups. Data were presented as the means $\pm \mathrm{SD}$ of at least three independent experiments. A two-tailed $\mathrm{P}<0.05$ was considered to be statistically significant.

\section{Abbreviations}

3'UTR : 3' untranslated region TNBC: triple negative breast cancer HUVECs : human umbilical vein endothelial cells EPLIN : epithelial protein lost in neoplasm

TCGA: The Cancer Genome Atlas

\section{Author contributions}

L Liang and Y Zan performed all the experiments and part of data analysis. L Zhao performed part of data analysis and drafted the manuscript. Q Zhu drafted the manuscript. J Ren and X Zhao designed the study and revised the manuscript.

\section{CONFLICTS OF INTEREST}

The authors declare no conflicts of interest.

\section{FUNDING}

This work was supported by the funding from Natural Science Foundation of Shaanxi Province (No. 2016JM8126). 


\section{REFERENCES}

1. Hanahan D, Weinberg RA. Hallmarks of cancer: the next generation. Cell. 2011; 144:646-74.

2. Ribatti D, Nico B, Ruggieri S, Tamma R, Simone G, Mangia A. Angiogenesis and antiangiogenesis in triplenegative breast cancer. Transl Oncol. 2016; 9:453-7.

3. Strickler JH, Hurwitz HI. Bevacizumab-based therapies in the first-line treatment of metastatic colorectal cancer. Oncologist. 2012; 17:513-24.

4. Sharma SG, Nanda S, Longo S. Anti-angiogenic therapy in renal cell carcinoma. Recent Patents Anticancer Drug Discov. 2010; 5:77-83.

5. Welker MW, Trojan J. Anti-angiogenesis in hepatocellular carcinoma treatment: current evidence and future perspectives. World J Gastroenterol. 2011; 17:3075-81.

6. Vasudev NS, Reynolds AR. Anti-angiogenic therapy for cancer: current progress, unresolved questions and future directions. Angiogenesis. 2014; 17:471-94.

7. Hao M, Zang M, Zhao L, Deng S, Xu Y, Qi F, An G, Qin Y, Sui W, Li F, Yang W, Li Z, Yi S, et al. Serum high expression of miR-214 and miR-135b as novel predictor for myeloma bone disease development and prognosis. Oncotarget. 2016; 7:19589-600. https://doi.org/10.18632/ oncotarget. 7319.

8. Ding Y, Ma Q, Liu F, Zhao L, Wei W. The potential use of salivary miRNAs as promising biomarkers for detection of cancer: a meta-analysis. PLoS One. 2016; 11:e0166303.

9. Landskroner-Eiger S, Moneke I, Sessa WC. miRNAs as modulators of angiogenesis. Cold Spring Harb Perspect Med. 2013; 3:a006643. https://doi.org/10.1101/cshperspect.a006643.

10. Deng Z, Qian J, Liu FQ, Lin J, Shao R, Yin JY, Tang Q, Zhang M, He L. Expression level of miR-93 in formalinfixed paraffin-embedded tissues of breast cancer patients. Genet Test Mol Biomark. 2014; 18:366-70.

11. Kawano M, Tanaka K, Itonaga I, Ikeda S, Iwasaki T, Tsumura H. microRNA-93 promotes cell proliferation via targeting of PTEN in osteosarcoma cells. J Exp Clin Cancer Res. 2015; 34:76.

12. Liang H, Wang F, Chu D, Zhang W, Liao Z, Fu Z, Yan X, Zhu H, Guo W, Zhang Y, Guan W, Chen X. miR-93 functions as an oncomiR for the downregulation of PDCD4 in gastric carcinoma. Sci Rep. 2016; 6:23772. https://doi. org/10.1038/srep23772.

13. Fang L, Du WW, Yang W, Rutnam ZJ, Peng C, Li H, O'Malley YQ, Askeland RW, Sugg S, Liu M, Mehta T, Deng Z, Yang BB. MiR-93 enhances angiogenesis and metastasis by targeting LATS2. Cell Cycle. 2012; 11: 4352-65.

14. Fang L, Deng Z, Shatseva T, Yang J, Peng C, Du WW, Yee AJ, Ang LC, He C, Shan SW, Yang BB. MicroRNA miR-93 promotes tumor growth and angiogenesis by targeting integrin- $\beta 8$. Oncogene. 2011; 30:806-21.

15. Fabbri E, Brognara E, Montagner G, Ghimenton C, Eccher A, Cantù C, Khalil S, Bezzerri V, Provezza L, Bianchi N, Finotti A, Borgatti M, Moretto G, et al. Regulation of IL-8 gene expression in gliomas by microRNA miR-93. BMC Cancer. 2015; 15:661.

16. Sanders AJ, Ye L, Mason MD, Jiang WG. The impact of EPLINa (Epithelial protein lost in neoplasm) on endothelial cells, angiogenesis and tumorigenesis. Angiogenesis. 2010; $13: 317-26$

17. Hu J, Xu J, Wu Y, Chen Q, Zheng W, Lu X, Zhou C, Jiao D. Identification of microRNA-93 as a functional dysregulated miRNA in triple-negative breast cancer. Tumor Biol. 2015; 36:251-8.

18. Collins RJ, Jiang WG, Hargest R, Mason MD, Sanders AJ. EPLIN: a fundamental actin regulator in cancer metastasis? Cancer Metastasis Rev. 2015; 34:753-64.

19. Hazarika S, Farber CR, Dokun AO, Pitsillides AN, Wang T, Lye RJ, Annex BH. MicroRNA-93 controls perfusion recovery following hind-limb ischemia by modulating expression of multiple genes in the cell cycle pathway. Circulation. 2013; 127:10.

20. Yang IP, Tsai HL, Hou MF, Chen KC, Tsai PC, Huang SW, Chou WW, Wang JY, Juo SH. MicroRNA-93 inhibits tumor growth and early relapse of human colorectal cancer by affecting genes involved in the cell cycle. Carcinogenesis. 2012; 33:1522-30.

21. Chu S, Liu G, Xia P, Chen G, Shi F, Yi T, Zhou H. miR93 and PTEN: key regulators of doxorubicin-resistance and EMT in breast cancer. Oncol Rep. 2017; 38:2401-7.

22. Crampton SP, Davis J, Hughes CC. Isolation of human umbilical vein endothelial cells (HUVEC). J Vis Exp. 2007; 183.

23. Starr TK, Scott PM, Marsh BM, Zhao L, Than BL, O’Sullivan MG, Sarver AL, Dupuy AJ, Largaespada DA, Cormier RT. A Sleeping Beauty transposon-mediated screen identifies murine susceptibility genes for adenomatous polyposis coli (Apc)-dependent intestinal tumorigenesis. Proc Natl Acad Sci U S A. 2011; 108:5765-70.

24. Sui C, Ma Q, Nan K, Xiao J, Suo A, Sha H, Zhao L. hSSTR2 expression and octreotide treatment reverses multidrug resistance of BxPC-3 human pancreatic cancer cells. Oncol Rep. 2009; 22:1391-6.

25. Than BL, Goos JA, Sarver AL, O'Sullivan MG, Rod A, Starr TK, Fijneman RJ, Meijer GA, Zhao L, Zhang Y, Largaespada DA, Scott PM, Cormier RT. The role of KCNQ1 in mouse and human gastrointestinal cancers. Oncogene. 2014; 33:3861-8. 